\title{
Traversable Wormholes and the Brouwer Fixed-Point Theorem
}

\author{
Peter K. F. Kuhfittig \\ Department of Mathematics, Milwaukee School of Engineering, Milwaukee, WI, USA \\ Email:kuhfitti@msoe.edu
}

How to cite this paper: Kuhfittig, P.K.F. (2020) Traversable Wormholes and the Brouwer Fixed-Point Theorem. Journal of Applied Mathematics and Physics, 8, 1263-1268.

https://doi.org/10.4236/jamp.2020.87096

Received: June 9, 2020

Accepted: July 6, 2020

Published: July 9, 2020

Copyright $\odot 2020$ by author(s) and Scientific Research Publishing Inc. This work is licensed under the Creative Commons Attribution International License (CC BY 4.0).

http://creativecommons.org/licenses/by/4.0/

\begin{abstract}
The Brouwer fixed-point theorem in topology states that for any continuous mapping $f$ on a compact convex set into itself admits a fixed point, i.e., a point $x_{0}$ such that $f\left(x_{0}\right)=x_{0}$. Under suitable conditions, this fixed point corresponds to the throat of a traversable wormhole, i.e., $b\left(r_{0}\right)=r_{0}$ for the shape function $b=b(r)$. The possible existence of wormholes can therefore be deduced from purely mathematical considerations without going beyond the existing physical requirements.
\end{abstract}

\section{Keywords}

Traversable Wormholes, Brouwer Fixed-Point Theorem

\section{Introduction}

Wormholes are handles or tunnels in spacetime connecting widely separated regions of our Universe or entirely different universes. Morris and Thorne [1] proposed the following line element for the wormhole spacetime:

$$
\mathrm{d} s^{2}=-\mathrm{e}^{2 \Phi(r)} \mathrm{d} t^{2}+\frac{\mathrm{d} r^{2}}{1-b(r) / r}+r^{2}\left(\mathrm{~d} \theta^{2}+\sin ^{2} \theta \mathrm{d} \phi^{2}\right),
$$

using units in which $c=G=1$. Here $\Phi=\Phi(r)$ is called the redshift function, which must be finite everywhere to prevent the appearance of an event horizon. The function $b=b(r)$ is called the shape function since it determines the spatial shape of the wormhole when viewed, for example, in an embedding diagram [1]. The spherical surface $r=r_{0}$ is the throat of the wormhole and is characterized by the following condition: $b\left(r_{0}\right)=r_{0}$. Mathematically speaking, $r=r_{0}$ is called a fixed point of the function $b=b(r)$ and will play a key role in our discussion. For a traversable wormhole, an important requirement is the flare-out 
condition $b^{\prime}\left(r_{0}\right)<1$; also, $b(r)<r$ for $r>r_{0}$. The flare-out condition can only be met by violating the null energy condition [NEC], which states that

$$
T_{\alpha \beta} k^{\alpha} k^{\beta} \geq 0
$$

for all null vectors $k^{\alpha}$, where $T_{\alpha \beta}$ is the stress-energy tensor. Matter that violates the NEC is called "exotic" in Ref. [1]. In particular, for the outgoing null vector $(1,1,0,0)$, the violation has the form

$$
T_{\alpha \beta} k^{\alpha} k^{\beta}=\rho+p_{r}<0 .
$$

Here $T_{t}^{t}=-\rho$ is the energy density, $T_{r}^{r}=p_{r}$ is the radial pressure, and $T_{\theta}^{\theta}=T_{\phi}^{\phi}=p_{t}$ is the lateral pressure. For completeness, let us also list the Einstein field equations:

$$
\begin{gathered}
\rho(r)=\frac{b^{\prime}}{8 \pi r^{2}}, \\
p_{r}(r)=\frac{1}{8 \pi}\left[-\frac{b}{r^{3}}+2\left(1-\frac{b}{r}\right) \frac{\Phi^{\prime}}{r}\right],
\end{gathered}
$$

and

$$
p_{t}(r)=\frac{1}{8 \pi}\left(1-\frac{b}{r}\right)\left[\Phi^{\prime \prime}-\frac{b^{\prime} r-b}{2 r(r-b)} \Phi^{\prime}+\left(\Phi^{\prime}\right)^{2}+\frac{\Phi^{\prime}}{r}-\frac{b^{\prime} r-b}{2 r^{2}(r-b)}\right] .
$$

The purpose of this paper is to make use of fixed-point theory to show that certain physical conditions imply the possible existence of traversable wormholes. To that end, we need the following special case of the Brouwer fixed-point theorem:

Theorem [2]. Let $f$ be a continuous function from a closed interval $[a, b]$ on the real line into itself. Then $f$ has a fixed point, i.e., there is a point $x_{0}$ such that $f\left(x_{0}\right)=x_{0}$.

A function that maps a set into itself is called a self-mapping.

\section{Some Consequences of the Brouwer Fixed-Point Theorem}

According to Ref. [3], the total mass-energy $M$ of an isolated star is well defined as long as one retains spherical symmetry. In Schwarzschild coordinates,

Total mass-energy inside radius

$$
r \equiv m(r)=\int_{0}^{r} 4 \pi\left(r^{\prime}\right)^{2} \rho\left(r^{\prime}\right) \mathrm{d} r^{\prime},
$$

where $\rho(r)$ is the energy density. Moreover, everywhere outside the star,

$$
m(r)=M \equiv \text { total mass-energy in the Newtonian limit. }
$$

We also have from Equation (4) that

$$
b(r)=\int_{0}^{r} 8 \pi\left(r^{\prime}\right)^{2} \rho\left(r^{\prime}\right) \mathrm{d} r^{\prime} .
$$

The line element (page 608 in Ref. [3]) is given by

$$
\begin{aligned}
\mathrm{d} s^{2} & =-\mathrm{e}^{2 \Phi(r)} \mathrm{d} t^{2}+\frac{\mathrm{d} r^{2}}{1-2 m(r) / r}+r^{2}\left(\mathrm{~d} \theta^{2}+\sin ^{2} \theta \mathrm{d} \phi^{2}\right) \\
& =-\left(1-\frac{2 M}{r}\right) \mathrm{d} t^{2}+\frac{\mathrm{d} r^{2}}{1-2 M / r}+r^{2}\left(\mathrm{~d} \theta^{2}+\sin ^{2} \theta \mathrm{d} \phi^{2}\right), \quad \text { for } r>R,
\end{aligned}
$$


where $R$ is the radius of the spherical star. According to Equations (7) and (9), $b(r)=2 m(r)$. With our wormhole spacetime in mind, a more convenient form of the line element is

$$
\mathrm{d} s^{2}=-\mathrm{e}^{\Phi(r)} \mathrm{d} t^{2}+\frac{\mathrm{d} r^{2}}{1-m(r) / r}+r^{2}\left(\mathrm{~d} \theta^{2}+\sin ^{2} \theta \mathrm{d} \phi^{2}\right), \quad \text { inside radius } r,
$$

and

$$
\mathrm{d} s^{2}=-\left(1-\frac{M}{r}\right) \mathrm{d} t^{2}+\frac{\mathrm{d} r^{2}}{1-M / r}+r^{2}\left(\mathrm{~d} \theta^{2}+\sin ^{2} \theta \mathrm{d} \phi^{2}\right), \quad \text { for } r>R,
$$

where $R$ is the radius of the star. Now $m(r)$ corresponds to $b(r)$ in line element (1).

To apply the Brouwer fixed-point theorem, we need to make use of the fact that every star has a dense core of radius $r=r_{1}$. For example, the average density of our sun is approximately $1.4 \mathrm{~g} / \mathrm{cm}^{3}$, while the density of the core is well over $100 \mathrm{~g} / \mathrm{cm}^{3}$. We will consider some other quantitative aspects in the next section.

Suppose $\rho(r)$ denotes the mass density of the star for $r>r_{1}$ and consider the mass

$$
m^{*}(r)=\int_{r_{1}}^{r} 4 \pi\left(r^{\prime}\right)^{2} \rho\left(r^{\prime}\right) \mathrm{d} r^{\prime} .
$$

Then $m^{*}\left(r_{1}\right)=0$ and the mapping

$$
m^{*}:\left[r_{1}, \infty\right) \rightarrow[0, \infty)
$$

is no longer a self-mapping. Let us therefore denote the mass of the spherical core of the star by $K(r)$ and its mass density by $\rho_{1}(r)$. Then $\rho_{1}(r)>\rho(r)$. Now consider a new mapping

$$
m(r)=\int_{r_{1}}^{r} 4 \pi\left(r^{\prime}\right)^{2} \rho\left(r^{\prime}\right) \mathrm{d} r^{\prime}+K\left(r_{1}\right),
$$

where $K\left(r_{1}\right)>r_{1}$. Since $m\left(r_{1}\right)=K\left(r_{1}\right)$, we see that $K\left(r_{1}\right)$ is indeed the mass of the core. Letting $r=R$ be the radius of the star, we now draw the important conclusion that $m(r)$ in Equation (13) maps the closed interval $\left[r_{1}, R\right]$ into itself, i.e.,

$$
\left[K\left(r_{1}\right), R^{*}\right] \subset\left[r_{1}, R\right]
$$

where

$$
R^{*}=m(R)=\int_{\eta_{1}}^{R} 4 \pi r^{2} \rho(r) \mathrm{d} r+K\left(r_{1}\right),
$$

provided that $R^{*} \leq R$. This conclusion can be illustrated graphically, as shown in Figure 1.

We also have

$$
m^{\prime}(r)=4 \pi r^{2} \rho(r) \ll 1,
$$

since $\rho(r)$ is very small in our geometrized units. So while $m(r)$ is an increasing function, $m^{\prime}(r)$ remains less than unity. 


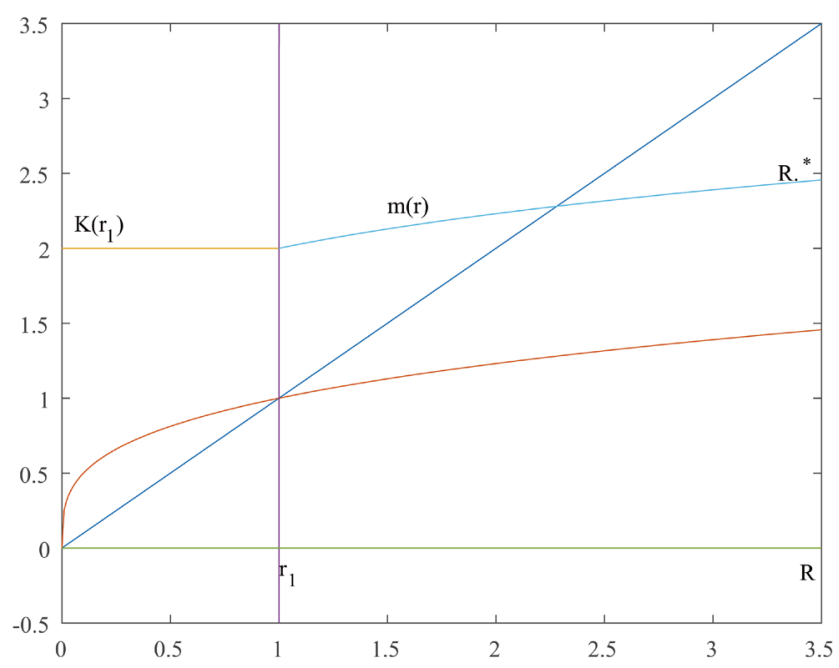

Figure 1. $m(r)$ maps the closed interval $\left[r_{1}, R\right]$ into itself.

We have seen that we obtain a self-mapping provided that $K\left(r_{1}\right)>r_{1}$. However, Equation (14) and Figure 1 show that the mass $K\left(r_{1}\right)$ of the core must not be excessively large.

Finally, from the Brouwer fixed-point theorem, we obtain (since $b(r) \equiv m(r)$ )

$$
b\left(r_{0}\right)=m\left(r_{0}\right)=\int_{r_{1}}^{r_{0}} 4 \pi r^{2} \rho(r) \mathrm{d} r+K\left(r_{1}\right)=r_{0} .
$$

By Equation (16), $b^{\prime}\left(r_{0}\right)=m^{\prime}\left(r_{0}\right)<1$, so that the flare-out condition is satisfied.

In the resulting wormhole spacetime, the region inside the throat $r=r_{0}$ is not part of the wormhole, but this region still contributes to the gravitational field. This can be compared to a thin-shell wormhole resulting from a Schwarzschild black hole [4]: while not part of the manifold, the black hole generates the underlying gravitational field.

As actual (quantified) example of the type of wormhole discussed is given in Ref. [5]. It is shown that for a typical neutron star, the possible formation of a wormhole requires a core of quark matter that is approximately $1 \mathrm{~m}$ in radius. (Quark matter is believed to exist at the center of neutron stars [6].) Qualitatively speaking, the conditions above apply to any star since, as already noted, stars are known to have dense cores.

\section{A Dark-Matter Background}

In the discussion of dark matter, several models have been proposed for the energy density. The best-known of these is the Navarro-Frenk-White model [7]

$$
\rho(r)=\frac{\rho_{s}}{\frac{r}{r_{s}}\left(1+\frac{r}{r_{s}}\right)^{2}},
$$

where $r_{s}$ is the characteristic scale radius and $\rho_{s}$ is the corresponding density. The Universal Rotation Curve [8] is given by 


$$
\rho(r)=\frac{\rho_{c} r_{c}^{3}}{\left(r+r_{c}\right)\left(r^{2}+r_{c}^{2}\right)},
$$

where $r_{c}$ is the core radius of the galaxy and $\rho_{c}$ is the central halo density. Another example is the King model whose energy density is given by [9]

$$
\rho(r)=\kappa\left(\frac{r^{2}}{r_{0}}+\lambda\right)^{\eta},
$$

where $\eta, \kappa, r_{0}$, and $\lambda$ are constants.

All of these models have a low energy density. So if $r_{1}$ is the radius of a star, then the star itself becomes the core since its energy density is much larger than that of the surrounding dark matter. Furthermore, since there is no outer boundary, we can choose $R$ large enough so that $m(r)$ in Equation (13) is a self-mapping. The existence of a fixed point now implies the possible existence of a wormhole in the dark-matter region.

\section{Conclusion}

A typical star has a dense spherical core. If $r=r_{1}$ denotes the radius of the core and $K\left(r_{1}\right)$ its mass, then $m(r)$, the effective mass of the star, is given by Equation (13). The function $m(r)$ satisfies the hypothesis of the Brouwer fixed-point theorem. The fixed point can be viewed as the radius of the throat of a traversable wormhole since $b\left(r_{0}\right)=m\left(r_{0}\right)=r_{0}$ and $b^{\prime}\left(r_{0}\right)=m^{\prime}\left(r_{0}\right)<1$. This result agrees with an earlier finding [5] showing that a typical neutron star requires a core of quark matter of radius $1 \mathrm{~m}$ for the possible existence of a wormhole. The above result can also be applied to a dark-matter setting by treating a star of radius $r=r_{1}$ as the core. So the possible existence of traversable wormholes follows directly from purely mathematical considerations without going beyond the physical requirements already in place.

\section{Conflicts of Interest}

The author declares no conflicts of interest regarding the publication of this paper.

\section{References}

[1] Morris, M.S. and Thorne, K.S. (1988) Wormholes in Spacetime and Their Use for Interstellar Travel: A Tool for Teaching General Relativity. American Journal of Physics, 56, 395-412. https://doi.org/10.1119/1.15620

[2] Brouwer, L.E.J. (1911) Über Abbildungen von Mannigfaltikeiten. Mathematische Annalen, 71, 97-115.

[3] Misner, C.W., Thorne, K.S. and Wheeler, J.A. (1973) Gravitation. W.H. Freeman and Company, New York, 603-608.

[4] Poisson, E. and Visser, M. (1995) Thin-Shell Wormholes: Linear Stability. Physical Review D, 52, 7318-7321. https://doi.org/10.1103/physrevd.52.7318

[5] Kuhfittig, P.K.F. (2013) Neutron Star Interiors and Topology Change. Advances in Mathematical Physics, 2013, Article ID: 630196. 
https://doi.org/10.1155/2013/630196

[6] Perez-Garcia, M.A., Silk, J. and Stone, J.R. (2010) Dark Matter, Neutron Stars, and Strange Quark Matter. Physical Review Letters, 105, Article ID: 141101. https://doi.org/10.1103/physrevlett.105.141101

[7] Navarro, J.F., Frenk, C.S. and White, S.D.M. (1996) The Structure of Cold Dark Matter Halos. Astrophysical Journal, 462, 563-575. https://doi.org/10.1086/177173

[8] Castignani, G., Fruscianti, N., Vernieri, D. and Salucci, P. (2012) The Density Profiles of Dark Matter Halos in Spiral Galaxies. Natural Science, 4, 265-270. https://doi.org/10.4236/ns.2012.45038

[9] King, I.R. (1972) Density Data and Emission Measure for a Model of the Coma Cluster. Astrophysical Journal, 174, L123. https://doi.org/10.1086/180963 